untersuchten Parameter keinen Sprung der Phasenverschiebung zwischen äußerem Magnetfeld und Bogenbewegung um $\Delta \psi=\pi$. Die Säule des Lichtbogens bewegt sich hier also in Richtung der Lorentz-Kraft, eine retrograde Bewegung tritt nicht ein. Messungen an einem 12 A-Bogen bei sonst ungeänderten Parametern hatten für den Druckbereich $50 \leqq p \leqq 500$ Torr ein ähnliches Ergebnis. Es folgt also, daß in dem untersuchten Parameterbereich keine retrograde Bewegung auftritt, deren Ursache in der Bogensäule selbst zu finden ist.
Die hier beschriebene Anordnung ist geeignet, weitere Bereiche der Parameter Druck, Bogenlänge, Magnetfeld, Stromstärke und Füllgas zu untersuchen und so $\mathrm{zu}$ entscheiden, ob eine retrograde Bewegung auf Grund eines Effektes in der Bogensäule für andere als die hier untersuchten Parameterbereiche möglich ist.

Herrn cand.-phys. H. Grosse-Wilde danke ich für seinen wertvollen Beitrag zum Aufbau der Apparatur.

\section{The Generalised Mean Square Amplitudes of Vibration for Substituted Ethylenes Vinylidene Halides and their Deuterates}

\author{
S. Jeyapandian and G. A. Savari RaJ \\ Department of Physics, St. Joseph's College \\ Tiruchirapalli-2, University of Madras, South India \\ (Z. Naturforsch. 26 a, 778-780 [1971] ; recived 17 January 1971)
}

For six vinylidene halides the generalised mean square amplitudes of displacements parallel and perpendicular to the bond for both bonded and non-bonded atom distances are calculated from spectroscopic data using the method of Morino and Hirato. The results are discussed and the mean amplitudes of these molecules $\left(\mathrm{H}_{2} \mathrm{C}=\mathrm{CX}_{2}\right)$ are compared with those from electron diffraction data for the similar molecules of the type $\mathrm{C}_{2} \mathrm{X}_{4}$.

\section{Introduction}

Recent developments in electron diffraction analysis of molecular structure require the knowledge of the generalised mean square amplitudes of vibration for bonded and nonbonded interatomic distances computed from spectroscopic data. The calculated values of mean square perpendicular amplitudes can be used not only to study the Bastiansen-Morino shrinkage effects for individual molecules, but also to evaluate the average structure of molecules in the ground vibrational state ${ }^{1}$. The mean square amplitudes of vibration for ethylene, ethylene- $\mathrm{d}_{4}$ and for some halogen substituted ethylenes have been already calculated ${ }^{1-5}$. In this paper we report the generalised mean square amplitudes of parallel and perpendicular displacements both for bonded and non-bonded atom distances for the vinylidene halides and their deuterates.

Reprints request to Dr. G. A. Savari RaJ, Department of Physics, St. Joseph's College, Tiruchirapalli-2, Tamil Nadu, South India.

1 K. Kuchitsu, J. Chem. Phys. 44, 906 [1966].

2 G. De Alti, V. Galasso, and G. Costa, Spectrochim. Acta 21, 649 [1965].

3 K. Venkateswaralu and S. Mariam, Czechoslov. J. Phys. B 16, 290 [1966].

\section{Molecular Model}

The Vinylidene halides and their deuterates belong to the $\mathrm{C}_{2 \mathrm{v}}$ point group with 12 normal modes of vibration distributed as $5 A_{1}+4 B_{1}+1 A_{2}+2 B_{2}$. The axes for vibrations are taken as follows: The $x$ axis is taken in the molecular plane and perpendicular to the line joining the equilibrium positions of the atoms; the $y$ axis is taken perpendicular to the molecular plane, and $z$ axis is taken along the line joining equilibrium positions of the atoms.

\section{Computations}

The generalised mean square parallel amplitude $\left\langle\Delta z^{2}\right\rangle$ and perpendicular amplitudes $\left\langle\Delta y^{2}\right\rangle$ and $\left\langle\Delta x^{2}\right\rangle$ and the mean cross products $\langle\Delta z \Delta x\rangle,\langle\Delta x \Delta y\rangle$ and $\langle\Delta y \Delta z\rangle$ are evaluated by the method of MORINO and Hirato ${ }^{1,6}$ using the formula,

$\left\langle A x^{2}\right\rangle=\left[D^{x}\left(M^{-1} B_{\mathrm{s}}{ }^{\prime}\right)\right]^{\prime}\left(L^{-1}\right)^{\prime}\left\langle Q^{2}\right\rangle L^{-1}\left[D^{x}\left(M^{-1} B_{\mathrm{s}}{ }^{\prime}\right)\right]$ with similar expressions for $\left\langle\Delta y^{2}\right\rangle$ and $\left\langle\Delta z^{2}\right\rangle$. The symbols have the same significance as in Ref. ${ }^{6}$. The elements of the diagonal matrix $\left\langle Q^{2}\right\rangle$ are given by

$$
\left\langle Q_{i}{ }^{2}\right\rangle=\frac{h}{8 \pi^{2} c \omega_{i}} \operatorname{coth}\left(\frac{h c \omega_{i}}{2 k T}\right)
$$

where $\omega_{i}$ is the wave number of the $i$-th normal vibrations.

A normal coordinate analysis for these molecules has already been made making use of Urey-Bradley force field, and the force constants have been adjusted by a suitable least square procedure so as to reproduce the fundamental frequencies mostly within one per cent error. The symmetry coordinates, the adjusted force

4 Y. Morino, K. Kuchitsu, and T. Shimanouchi, J. Chem. Phys. 20, 726 [1952].

5 S. J. Cyvin, Czech. J. Phys. B 20, 464 [1970].

6 Y. Morino and E. Hirota, J. Chem. Phys. 23, 737 [1955].

7 S. Jeyapandian and G. A. Savari Raj, J. Mol. Struct. (in press).

8 E. B. Wilson, Jr., J. Chem. Phys. 9, 76 [1941]. 
Table 1. The generalised mean square amplitudes of vibration of vinylidene halides and their deuterates (in $\AA^{2}$ ) at $0{ }^{\circ} \mathrm{K}$ and $298{ }^{\circ} \mathrm{K}$ (* non bonded atom pair).

\begin{tabular}{|c|c|c|c|c|c|c|c|c|c|}
\hline \multirow[t]{2}{*}{ Molecule } & \multirow[t]{2}{*}{ Atom pair } & \multicolumn{2}{|c|}{ Parallel $\left\langle\Delta z^{2}\right\rangle$} & \multicolumn{2}{|c|}{$\begin{array}{c}\text { (a) } \mathrm{H}_{2} \mathrm{C}=\mathrm{CF}_{2} \\
\text { Perpendicular }\left\langle\Delta x^{2}\right\rangle\end{array}$} & \multicolumn{2}{|c|}{ Perpendicular $\left\langle\Delta y^{2}\right\rangle$} & \multicolumn{2}{|c|}{$\begin{array}{c}\text { Mean cross products } \\
\langle\Delta z \Delta x\rangle\end{array}$} \\
\hline & & at $0^{\circ} \mathrm{K}$ & at $298^{\circ} \mathrm{K}$ & at $0^{\circ} \mathrm{K}$ & at $298{ }^{\circ} \mathrm{K}$ & at $0{ }^{\circ} \mathrm{K}$ & at $298^{\circ} \mathrm{K}$ & at $0^{\circ} \mathrm{K}$ & at $298^{\circ} \mathrm{K}$ \\
\hline \multirow[t]{5}{*}{$\mathrm{H}_{2} \mathrm{C}=\mathrm{CF}_{2}$} & $\mathrm{C}=\mathrm{C}$ & 0.001645 & 0.001667 & 0.003018 & 0.003503 & 0.002842 & 0.003089 & 0 & 0 \\
\hline & $\mathrm{C}-\mathrm{F}$ & 0.002394 & 0.002675 & 0.002091 & 0.002181 & 0.000298 & 0.000397 & -.000275 & -.000410 \\
\hline & $\mathrm{C}-\mathrm{H}$ & 0.013332 & 0.013900 & 0.009008 & 0.009221 & 0.037283 & 0.040137 & .004774 & .005144 \\
\hline & $F \ldots F *$ & 0.001915 & 0.002399 & 0.002437 & 0.002661 & 0.000163 & 0.000174 & 0 & 0 \\
\hline & $\mathrm{H} \ldots \mathrm{H}$ & 0.031339 & 0.033674 & 0.012679 & 0.015563 & 0.044074 & 0.046994 & 0 & 0 \\
\hline \multirow[t]{6}{*}{$\mathrm{D}_{2} \mathrm{C}=\mathrm{CF}_{2}$} & $\mathrm{C}=\mathrm{C}$ & 0.001638 & 0.001654 & 0.002859 & 0.003317 & 0.004395 & 0.004805 & 0 & 0 \\
\hline & $C-F$ & 0.002482 & 0.002857 & 0.002121 & 0.002240 & 0.002986 & 0.003572 & -.000539 & -.000528 \\
\hline & $C-D$ & 0.011654 & 0.012556 & 0.004928 & 0.006695 & 0.013841 & 0.015912 & 0.005859 & 0.004959 \\
\hline & $\mathrm{F} \ldots \mathrm{F}$ & 0.002333 & 0.003165 & 0.002452 & 0.002676 & 0.000469 & 0.000557 & 0 & 0 \\
\hline & D...D & 0.021420 & 0.024864 & 0.010824 & 0.109189 & 0.031659 & 0.037558 & 0 & 0 \\
\hline & \multicolumn{9}{|c|}{ (b) $\mathrm{H}_{2} \mathrm{C}=\mathrm{CCl}_{2}$} \\
\hline \multirow[t]{5}{*}{$\mathrm{H}_{2} \mathrm{C}=\mathrm{CCl}_{2}$} & $\mathrm{C}=\mathrm{C}$ & 0.001744 & 0.001759 & 0.003776 & 0.004663 & 0.001825 & 0.002052 & 0 & 0 \\
\hline & $\mathrm{C}-\mathrm{Cl}$ & 0.002593 & 0.003364 & 0.002449 & 0.002902 & 0.000147 & 0.000152 & -.000183 & -.000563 \\
\hline & $\mathrm{C}-\mathrm{H}$ & 0.014097 & 0.015030 & 0.009287 & 0.009634 & 0.042884 & 0.049216 & .005245 & .005799 \\
\hline & $\mathrm{Cl} \ldots \mathrm{Cl}$ & 0.000781 & 0.001073 & 0.002777 & 0.004294 & 0.000062 & 0.000067 & 0 & 0 \\
\hline & H...H & 0.033719 & 0.037253 & 0.015376 & 0.015569 & 0.046805 & 0.050319 & 0 & 0 \\
\hline \multirow[t]{5}{*}{$\mathrm{D}_{2} \mathrm{C}=\mathrm{CCl}_{2}$} & $\mathrm{C}=\mathrm{C}$ & 0.001748 & 0.001938 & 0.004044 & 0.005233 & 0.003802 & 0.004195 & 0 & 0 \\
\hline & $\mathrm{C}-\mathrm{Cl}$ & 0.002659 & 0.003492 & 0.002726 & 0.003526 & 0.001957 & 0.002097 & -.000217 & -.000614 \\
\hline & $C-D$ & 0.012446 & 0.014332 & 0.006798 & 0.007316 & 0.021495 & 0.026820 & 0.006339 & 0.007268 \\
\hline & Ci.... & 0.000977 & 0.001468 & 0.002769 & 0.004278 & 0.000169 & 0.000204 & 0 & 0 \\
\hline & \multicolumn{9}{|c|}{ (c) $\mathrm{H}_{2} \mathrm{C}=\mathrm{CBr}_{2}$} \\
\hline \multirow{5}{*}{$\mathrm{H}_{2} \mathrm{C}=\mathrm{CBr}_{2}$} & $\mathrm{C}=\mathrm{C}$ & 0.001827 & 0.001873 & 0.004268 & 0.005499 & 0.002533 & 0.002865 & 0 & 0 \\
\hline & $\mathrm{C}-\mathrm{Br}$ & 0.002752 & 0.004534 & 0.002809 & 0.004126 & 0.000303 & 0.000353 & -.0002117 & -.000965 \\
\hline & $\mathrm{C}-\mathrm{H}$ & 0.014353 & 0.015337 & 0.009280 & 0.009901 & 0.045197 & 0.054233 & .005379 & .006080 \\
\hline & $\mathrm{Br} \ldots \mathrm{Br}$ & 0.000204 & 0.0003078 & 0.001880 & 0.004282 & 0.000013 & 0.000014 & 0 & 0 \\
\hline & H...H & 0.034767 & 0.039518 & 0.015268 & 0.015289 & 0.044630 & 0.048313 & 0 & 0 \\
\hline \multirow[t]{5}{*}{$\mathrm{D}_{2} \mathrm{C}=\mathrm{CBr}_{2}$} & $\mathrm{C}=\mathrm{C}$ & 0.001793 & 0.001794 & 0.004063 & 0.005385 & 0.002895 & 0.003343 & 0 & 0 \\
\hline & $\mathrm{C}-\mathrm{Br}$ & 0.002682 & 0.004602 & 0.002910 & 0.004067 & 0.002658 & 0.002853 & -.000139 & -.000867 \\
\hline & $C-D$ & 0.010249 & 0.011893 & 0.006829 & 0.007285 & 0.023343 & 0.030123 & 0.003863 & 0.005116 \\
\hline & $\mathrm{Br} \ldots \mathrm{Br}$ & 0.000261 & 0.000398 & 0.001872 & 0.004242 & 0.000040 & 0.000049 & 0 & 0 \\
\hline & H...H & 0.033973 & 0.052835 & 0.010964 & 0.010976 & 0.034025 & 0.041618 & 0 & 0 \\
\hline
\end{tabular}

constants and the calculated vibrational frequencies for all these molecules excepting Vinylidene bromide and its deuterate are given in one of our previous papers ${ }^{7}$. The $L^{-1}$ matrix elements are computed using the standard methods ${ }^{8}$. The computed values of the generalised mean square amplitudes for bonded as well as non-bonded atom distances at temperatures $0{ }^{\circ} \mathrm{K}$ and at $298^{\circ} \mathrm{K}$ are given in Table 1 .

\section{Results and Discussions}

There are no experimental values available for Vinylidene halide to compare our calculated values with. However, KUCHITsU ${ }^{1}$ has calculated the generalised mean square amplitudes for $\mathrm{C}_{2} \mathrm{H}_{4}$ and $\mathrm{C}_{2} \mathrm{D}_{4}$; and $\mathrm{CY}$ viN ${ }^{5}$ for $\mathrm{C}_{2} \mathrm{~F}_{4}, \mathrm{C}_{2} \mathrm{Cl}_{4}, \mathrm{C}_{2} \mathrm{Br}_{4}$ and $\mathrm{C}_{2} \mathrm{I}_{4}$. The values for $\mathrm{C}=\mathrm{C}, \mathrm{C}-\mathrm{H}, \mathrm{C}-\mathrm{D}, \mathrm{C}-\mathrm{F}, \mathrm{C}-\mathrm{Cl}, \mathrm{C}-\mathrm{Br}$ obtained by these authors agree well, if the change in the atomic environment is borne in mind, with those in $\mathrm{H}_{2} \mathrm{C}=\mathrm{CF}_{2}$, $\mathrm{H}_{2} \mathrm{C}=\mathrm{CCl}_{2}$ and $\mathrm{H}_{2} \mathrm{C}=\mathrm{CBr}_{2}$ obtained by us. Further it is of interest to compare the available electron diffraction data for the mean amplitudes of vibration for the above mentioned bonded distances of $\mathrm{C}_{2} \mathrm{X}_{4}$ with those of $\mathrm{C}_{2} \mathrm{H}_{2} \mathrm{X}_{2}$ type. As seen from the Table 2 the mean amplitudes of $\mathrm{C}-\mathrm{F}, \mathrm{C}-\mathrm{Cl}, \mathrm{C}-\mathrm{Br}$, of $\mathrm{C}_{2} \mathrm{H}_{2} \mathrm{X}_{2}$ slightly vary with those of $\mathrm{C}_{2} \mathrm{X}_{4}$ molecules while the mean amplitudes of $\mathrm{C}=\mathrm{C}$ in both types of molecules are practically the same as expected.

All mean cross products $\langle\Delta z \Delta y\rangle$ and $\left\langle\Delta x \Delta y^{\prime}\right\rangle$ vanish because of symmetry and planarity conditions assumed. It is found that the parallel mean square amplitudes increase with the substitution of higher members of the halogen group. This may be due to the decrease in the electronegativity of the atoms concerned ${ }^{\boldsymbol{\theta}}$. The distances in which $\mathrm{H}$ atoms are involved have larger values for mean square amplitudes; but when $\mathrm{H}$ is substituted by $\mathrm{D}$, the mean square ampli-

9 K. Venkateswaralu et al., Indian J. Pure Appl. Phys. 7, 279 [1969]. 
Table 2. Mean amplitude of vibration ( $\AA$ units).

\begin{tabular}{lllll}
\hline & \multicolumn{2}{c}{ Bond } \\
Molecule & Nature Distance & $\begin{array}{c}\text { Mean } \\
\text { ampli- } \\
\text { tude }\end{array}$ \\
\hline \multicolumn{4}{c}{} \\
& \multicolumn{4}{c}{ Remarks } \\
$\mathrm{C}_{2} \mathrm{~F}_{4}$ & $\mathrm{C}=\mathrm{C}$ & 1.31 & .0403 & Calculated * \\
$\mathrm{C}_{2} \mathrm{H}_{2} \mathrm{~F}_{2}$ & $\mathrm{C}=\mathrm{C}$ & 1.311 & .0408 & Calculated (present work) \\
$\mathrm{C}_{2} \mathrm{~F}_{4}$ & $\mathrm{C}-\mathrm{F}$ & 1.31 & .0560 & Experimental ** \\
$\mathrm{C}_{2} \mathrm{H}_{2} \mathrm{~F}_{2}$ & $\mathrm{C}-\mathrm{F}$ & 1.321 & .0517 & Calculated (present work) \\
$\mathrm{C}_{2} \mathrm{Cl}_{4}$ & $\mathrm{C}=\mathrm{C}$ & 1.354 & .0437 & Calculated * \\
$\mathrm{C}_{2} \mathrm{H}_{2} \mathrm{Cl}_{2}$ & $\mathrm{C}=\mathrm{C}$ & 1.380 & .0419 & Calculated (present work) \\
$\mathrm{C}_{2} \mathrm{Cl}_{4}$ & $\mathrm{C}-\mathrm{Cl}$ & 1.718 & .0480 & Experimental *** \\
$\mathrm{C}_{2} \mathrm{H}_{2} \mathrm{Cl}_{2}$ & $\mathrm{C}-\mathrm{Cl}$ & 1.69 & .0579 & Calculated (present work) \\
$\mathrm{C}_{2} \mathrm{Br}_{4}$ & $\mathrm{C}=\mathrm{C}$ & 1.362 & .0435 & Calculated * \\
$\mathrm{C}_{2} \mathrm{H}_{2} \mathrm{Br}_{2}$ & $\mathrm{C}=\mathrm{C}$ & 1.31 & .0433 & Calculated (present work) \\
$\mathrm{C}_{2} \mathrm{Br}_{4}$ & $\mathrm{C}-\mathrm{Br}$ & 1.881 & .0430 & Experimental *** \\
$\mathrm{C}_{2} \mathrm{H}_{2} \mathrm{Br}_{2}$ & $\mathrm{C}-\mathrm{Br}$ & 1.900 & .0673 & Calculated (present work) \\
\hline
\end{tabular}

tudes are not very much altered for almost all the atom pairs in all these molecules. With the increase of temperature the mean square amplitudes increase more for the non-bonded distances than for the bonded distances.

\section{Acknowledgement}

One of us (S. J.) is grateful to the University Grants Commission (New Delhi) for the award of a Junior Research Fellowship in order to carry out these investigations. We are grateful to Dr. K. Ramasamy, Reader in Spectroscopy, Annamalai University, for his helpful suggestions.

* S. J. Cyvin, Czech. J. Phys. B 20, 464 [1970].

** I. L. Karle and J. J. Karle, J. Chem. Phys. 18, 963 [1950].

*** T. G. Strand, Acta Chem. Scand. 21, 2111 [1967].

\section{Zinndioxidschichten auf stabilisiertem Zirkon- dioxid als Kathoden in Hochtemperatur- Brennstoffzellen}

\author{
R. BÖHM und H. KLeinschmager \\ Zentrales Forschungslaboratorium (ZFL) der BBC AG \\ Heidelberg \\ (Z. Naturforsch. 26 a, $780-782$ [1971] ; eingegangen am 25. Februar 1971)
}

Durch "Chemical Vapour Deposition" auf porös aufgerauhtem Träger aus stabilisiertem $\mathrm{ZrO}_{2}$ abgeschiedene $\mathrm{SnO}_{2}$-Schichten mit mehr als 8 At.-Proz. Sb können als Kathoden in Hochtemperatur-Brennstoffzellen bei $1000{ }^{\circ} \mathrm{C}$ verwendet werden; die Materialkombination übersteht Temperaturzyklen zwischen Betriebs- und Zimmertemperatur ohne Beeinträchtigung der Haftfestigkeit oder der elektrischen Funk. tion.

Über halbleitende Zinndioxidschichten mit AntimonDotierung zur Herabsetzung ihres elektrischen Widerstandes wurde schon mehrfach berichtet ${ }^{1-5}$; auch wurden derartige $\mathrm{SnO}_{2}$-Filme bereits als Kathoden in Hochtemperatur-Brennstoffzellen (HTBZ-n) eingesetzt ${ }^{6}$. Diese Filme werden meist durch Hydrolyse von Zinn(IV). chlorid und Antimon(III) chlorid an einem auf 500 bis $900{ }^{\circ} \mathrm{C}$ vorgeheizten Substrat abgeschieden. So auf Borsilikatglas erzeugte Schichten besitzen maximale Kaltleitfähigkeit, verbunden mit positivem Widerstands-

1 J. McKinney Mochel u. E. M. Guyer, Dt. Pat. Nr. 908882 vom 12. April 1954, Corning Glass Works, Corning, N. Y. (USA).

A. FisCher, Z. Naturforsch. 9 a, 508 [1954].

3 R. GLANG, Dissertation, TH Darmstadt 1955.

4 V. K. Miloslavskir, Opt. i Spektr. 7, 246 [1959].

5 K. Ishiguro, T. Sasaki, T. Arai u. J. Imai, J. Phs. Soc. Japan 13, 296 [1958].
Temperatur-Koeffizienten, bei einem Sb-Anteil von ca. 1 At.-Prozent ${ }^{1}$. Eine Schicht ähnlichen Sb-Gehalts auf einem Zirkondioxid-Träger, bei $1000{ }^{\circ} \mathrm{C}$ als Kathode einer HTBZ eingesetzt, hob sich beim anschließenden Abkühlen auf $600{ }^{\circ} \mathrm{C}$ von der Unterlage ab. Als Ursache wurde eine elektrochemische, Bindungen zerstörende Reaktion vermutet ${ }^{6}$. Eine Verbesserung dieser unzureichenden Haftung wäre Voraussetzung für die Verwendbarkeit von Zinnoxid als Kathodenmaterial; wegen der relativen Preisgünstigkeit des Werkstoffs und der guten Leitfähigkeit (ca. $1 \cdot 10^{3} \Omega^{-1} \mathrm{~cm}^{-1}$ bei $1000{ }^{\circ} \mathrm{C}$ in Luft), die sich andernfalls nur mit teuereren Materialien auf Indium- oder Praseodym-Basis erreichen ließe ${ }^{6,7}$, stellten wir uns im Rahmen eines größeren, die Entwicklung einer HTBZ-n-Batterie beinhaltenden Projekts die oben skizzierte Aufgabe.

Die Zähigkeit von $\mathrm{SnO}_{2}$-Schichten läßt sich durch Erhöhen der Sb-Dotierung steigern ${ }^{1}$, bei allerdings gleichzeitig abnehmender (Kalt-) Leitfähigkeit. Da aber mit wachsendem Sb-Gehalt der Widerstands-TemperaturKoeffizient das Vorzeichen wechseln kann ${ }^{2,3}$ und jener auch vom thermischen Ausdehnungskoeffizienten des Substrates abhängt ${ }^{2}$, erscheint es möglich, bei höheren Sb-Gehalten $\mathrm{SnO}_{2}$-Filme verbesserter thermisch-mechanischer Beanspruchbarkeit zu erhalten, deren Leitfähigkeit bei $1000{ }^{\circ} \mathrm{C}$ gleichwohl ausreicht, den in einer HTBZ erzeugten Strom ohne unzulässig hohen Spannungsverlust abzuführen. Bei Verwendung von $\mathrm{ZrO}_{2}$ -

6 E. F. Sverdrup, D. H. Archer u. A. D. Glasser, 5th Biennial Fuel Cell Symposium, Sept. 12-14, 1967, in: Fuel Cell-Systems II (Adv. Chem. Ser. 90), Amer. Chem. Soc., Washington, D. C., 1969.

7 C. S. Tedmon jR., H. S. Spacil u. S. P. Mitoff, J. Electrochem. Soc. 116 (9), 1170 [1969]. 